\title{
PTEN loss and PSGR overexpression promote cancer progression
}

The development of a new mouse model by Rodriguez et al. has provided insights into the synergistic action of prostatespecific G-protein-coupled receptor (PSGR) overexpression and phosphatase and tensin homologue (PTEN) loss in the progression of prostate cancer.

\section{4 ...a synergistic mechanism between PTEN and PSGR in prostate cancer... 77}

PTEN is the most frequently deleted tumour-supressor gene in prostate cancer and its loss predicts poor prognosis and progression to a castration-resistant phenotype. Rodriguez and colleagues observed that PTEN was deleted or mutated in $21 \%$ of prostate cancers according to TCGA analysis. Conversely, PSGR is overexpressed in prostate tumours and expression levels correlate with cancer progression; when the team investigated genomic data sets they found that four of 10 displayed PSGR upregulation.
They then investigated how these two lesions collaborate to enhance prostate cancer development, creating a bigenic mouse model that overexpresses human PSGR with a conditional Pten knockout. Analysis at 12 months showed that the prostates of these double-mutant mice had a deformed structure and occlusion of the adjacent seminal vesicles. Ki67 staining revealed increased cell proliferation in prostate tissue. Invasive prostate tumours that featured Akt activation, infiltration by inflammatory cells and loss of E-cadherin had also developed. Further study revealed that stromal androgen receptor (AR) expression was increased. This increase might indicate that nuclear factor $\kappa$-lightchain-enhancer of activated B cells (NF- $\kappa \mathrm{B}$ ) activity is upregulated as a consequence of PSGR overexpression, as it regulates stromal AR expression. These data suggest the possibility of a synergistic mechanism between PTEN and PSGR in prostate cancer progression.

In vitro, PSGR overexpression affected oncogenic properties of cells directly and indirectly. PSGR-overexpressing LNCaP cells (which have an inactivating mutation in PTEN) showed increased proliferation, whereas LNCaP cells with PSGR knockdown showed decreased proliferation and cell migration. Coculturing $\mathrm{LNCaP}$ cells that overexpress PSGR with stromal WPMY-1 cells and Raw264.7 macrophages induced stromal AR expression and a further increase in PSGR, suggesting that intercellular crosstalk can occur.

These results suggest that loss of Pten and overexpression of PSGR, which occur together in the majority of prostate cancers, act synergistically to accelerate tumour development. A new model is also described that will enable investigation of the collaboration between these two lesions in the context of prostate cancer.

Louise Stone

Original article Rodriguez, M. et al. Prostate-specific G-protein-coupled receptor collaborates with loss of PTEN to promote prostate cancer progression. Oncogene doi:10.1038/onc.2015.170 